\title{
The Relationship Between Earlier Onset of Natural Menopause and Elevated Urinary Albumin-Creatinine Ratio in Postmenopausal Chinese Women
}

This article was published in the following Dove Press journal:

Diabetes, Metabolic Syndrome and Obesity: Targets and Therapy

\author{
Mingyan Yao ${ }^{1,2, *}$ \\ Hongzhou Liu',* \\ Bing Li \\ Yang Liu' \\ Yiming Mu' \\ 'Department of Endocrinology, Chinese \\ People's Liberation Army General \\ Hospital, Beijing, 100853, People's \\ Republic of China; ${ }^{2}$ Department of \\ Endocrinology, Baoding NO.I Central \\ Hospital, Baoding, 07I000, People's \\ Republic of China
}

*These authors contributed equally to this work
Correspondence: Yiming Mu

Department of Endocrinology, Chinese People's Liberation Army General Hospital, No. 28 Fuxing Road, Beijing, 100853, People's Republic of China Email muyiming@30Ihospital.com.cn

\begin{abstract}
Purpose: There is a close correlation between menopausal age and cardiovascular disease (CVD) risk. Some research suggests that this risk is attributable to an elevated urinary albumin-creatinine ratio (UACR), but further work is needed to explore the link between UACR and age at time of menopause.

Patients and Methods: Data analyzed in the present study were derived from seven regional centers participating in the REACTION study. A total of 21,672 postmenopausal women met with our study inclusion and exclusion criteria, and were split into three groups based upon their age at onset of natural menopause. A UACR $\geq 30 \mathrm{mg} / \mathrm{g}$ was the primary outcome measure for this study. Odds ratios (ORs) and 95\% confidence intervals (CIs) were calculated with a logistic regression approach.
\end{abstract}

Results: Relative to women who were $46-50$ years old at time of natural menopause, those with an earlier onset of menopause ( $\leq 45$ years) exhibited an increased risk of UACR elevation following adjustment for confounding variables (OR: 1.18, 95\% CI: 1.04-1.33), whereas the opposite was true for women with a later age of menopause onset ( $>50$ years) (OR: 0.86 , 95\% CI: $0.78-0.94$ ). For every 1-year delay in the onset of menopause, UACR risk fell by $3 \%$ (OR: $0.97,95 \%$ CI: $0.96-0.98)$.

Conclusion: In summary, early menopause ( $\leq 45$ years old) was linked to a higher risk of UACR elevation in postmenopausal women. However, further work will be needed to understand the mechanistic basis for these findings.

Keywords: age, urinary albumin-creatinine ratio, menopause

\section{Introduction}

Menopause is an important transitional period for women corresponding to the end of their reproductive lifespan. Age at time of natural menopause is a potential indicator of overall health status and somatic aging, with considerable variability among individuals. ${ }^{1}$ Studies of Western populations have shown that approximately $10 \%$ of women undergo menopause prior to the age of 45 , resulting in a significantly decreased premenopausal estrogen exposure duration relative to women with later-onset menopause. ${ }^{2,3}$ Muka et $\mathrm{al}^{4}$ conducted a meta-analysis which revealed an elevated risk of cardiovascular disease (CVD) and mortality among women who undergo early-onset menopause $(<45$ years $)$. 
Metabolic diseases can have a profound negative impact on kidney function, and chronic kidney disease (CKD) can drive the development of anemia, altered bone metabolism, cognitive disorder, and CVD., Microalbuminuria (MAU), in particular, is a sensitive biomarker of early renal vascular damage and CVD risk. ${ }^{7-10}$ The urinary albumin-creatinine ratio (UACR) can be used to monitor the rate of urinary albumin excretion, and there is some evidence that elevated UACR is linked to elevated cardiovascular mortality rates. ${ }^{11}$ Furthermore, in late-onset type 2 diabetes mellitus (T2DM) patients, each $3.01 \mathrm{mg} / \mathrm{g}(0.4 \mathrm{mg} / \mathrm{mmol})$ rise in UACR has been shown to be linked to a $5.9 \%$ rise in the risk of cardiovascular events. ${ }^{12}$ While UACR is closely linked to CVD risk, whether age of natural menopause onset is linked to increases in UACR remains to be established.

As such, the present large cross-sectional analysis was designed to evaluate the association between UACR and age at natural menopause among different subgroups of postmenopausal women in China.

\section{Patients and Methods Study Participants}

This cross-sectional analysis was conducted as a part of the ongoing longitudinal REACTION study, which aims to evaluate relationships between prediabetes, T2DM, and cancer rates in a Chinese population. ${ }^{13}$ In total, 47,808 participants $>40$ years of age were recruited to this study from seven regional centers (Dalian, Guangzhou, Zhengzhou, Lanzhou, Luzhou, Wuhan, and Shanghai), of whom 33,129 were female $(69.30 \%)$. Of these women, 11,457 were excluded from this analysis as (1) they were premenopausal ( $\mathrm{n}=6675$ ); (2) they did not have available menopause-related data $(\mathrm{n}=2252)$; or (3) they had undergone iatrogenic or unnatural menopause $(n=2190$; Figure 1). In addition, women diagnosed with primary kidney diseases (kidney stones [ $\mathrm{n}=8]$, nephrotic syndrome [ $\mathrm{n}=14]$, chronic kidney disease [ $\mathrm{n}=198]$, and other kidney diseases $[n=120]$ ) were excluded, resulting in the inclusion of 21,672 total women in the final analytical cohort (Figure 1). The Committee on Human Research at RuiJin Hospital affiliated with the School of Medicine, Shanghai Jiao Tong University approved the present study, which was consistent with the Declaration of Helsinki. All patients provided informed consent to participate.

\section{Data Collection}

A standardized approach was used by trained researchers to collect all study data. Study participants completed a questionnaire regarding their age, sex, education level, medical history, medication usage, menopause status, and behavior (sleep quality, physical activity, and smoking and drinking status). Patients were instructed to take off their shoes, coats, and hats prior to the measurement of weight and height. Waist circumference (WC) was measured at the horizontal level of the midpoint of the ligature between the anterior superior spine and the inferior margin of the twelfth rib. Hip circumference (HC) was defined at the horizontal level of the most protruding part of the hip. Subject blood pressure (BP) was assessed thrice at 1-minute intervals after a 5-minute rest period and averaged together. Venous blood was obtained after an overnight fast ( $\geq 10 \mathrm{~h}$ ). Oral glucose tolerance tests were conducted using $75 \mathrm{~g}$ or $100 \mathrm{~g}$ of steamed bread for patients without T2DM and with T2DM, respectively. An automated enzymatic approach was used to measure fasting blood glucose (FBG), $2 \mathrm{~h}$ post-load blood glucose (PBG), triglycerides (TG), high-density lipoprotein cholesterol (HDL-C), lowdensity lipoprotein cholesterol (LDL-C), haemoglobin A1c (HbA1c), alanine transferase (ALT), aspartate transferase (AST), gamma-glutamyl transferase (GGT), and serum creatinine $(\mathrm{Cr})$ at appropriate regional centers. Levels of urinary creatinine and albumin were analyzed using sterile first-void urine samples obtained in the morning.

\section{Study Variable Definitions}

Patient body mass index (BMI) was defined by dividing patient weight $(\mathrm{kg})$ by the square of their height $(\mathrm{m})$. The waist-to-hip ratio was calculated by dividing WC by HC. Patient estimated glomerular filtration rate (eGFR) was defined based upon the Chronic Kidney Disease Epidemiology Collaboration (CKD-EPI) criteria. ${ }^{14}$ Participant educational level was classified as being low (illiteracy or primary education only), intermediate (junior to senior secondary education or vocational education), or high (higher vocational or university education). Sleep quality was classified as being either poor or good. Hormone therapy was defined as a history of the use of oral contraceptives and/or hormone replacement therapy (estrogen vs estrogen and progesterone). Participant alcohol intake and smoking status were defined based upon whether they never drank/smoked, were current smokers/ drinkers, or had quit drinking/smoking. T2DM was 


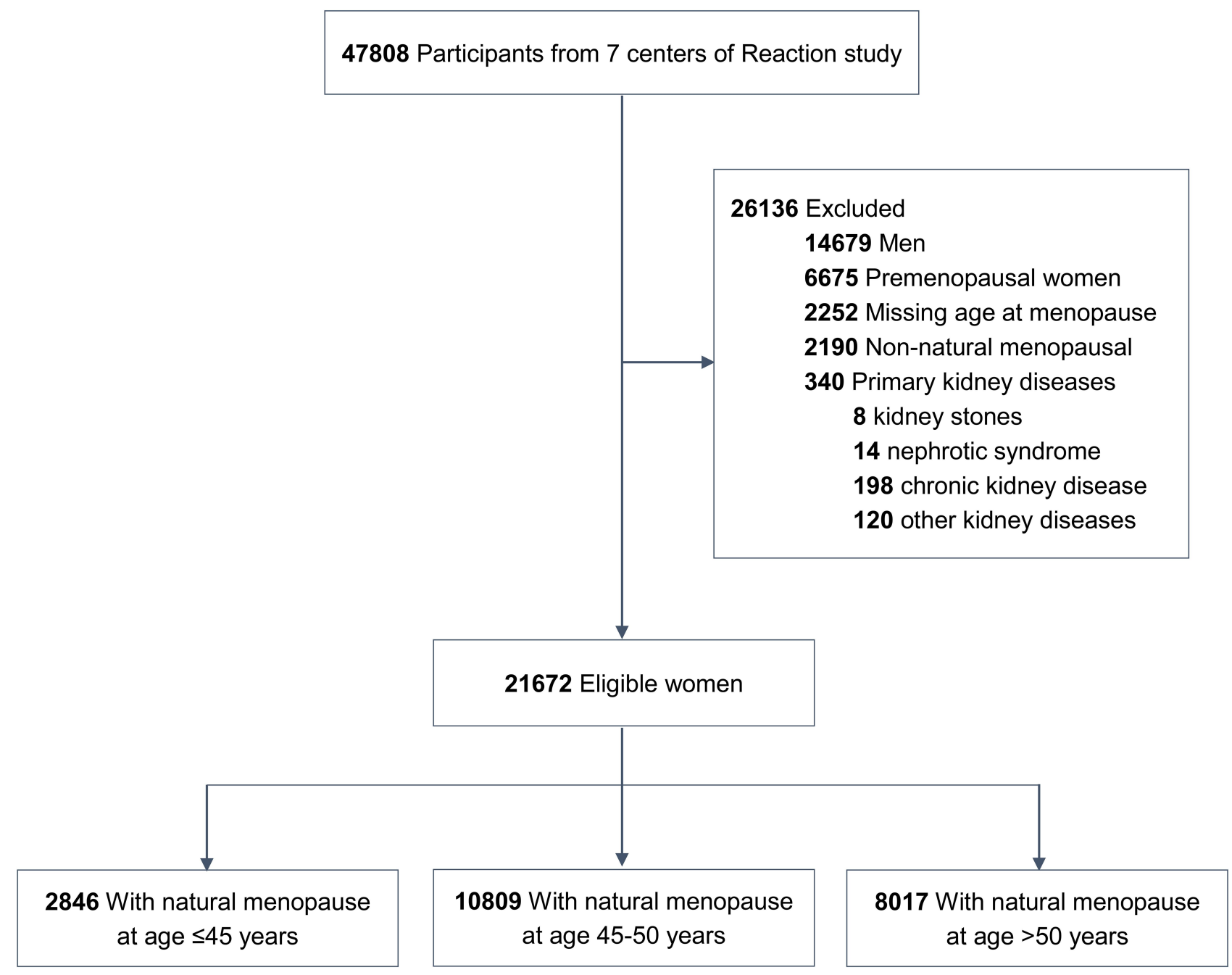

Figure I Flowchart of participants from the REACTION Study.

diagnosed as per WHO guidelines as a history of T2DM or an $\mathrm{FBG} \geq 7.0 \mathrm{mmol} / \mathrm{L}$ or $\mathrm{PBG} \geq 11.1 \mathrm{mmol} / \mathrm{L}$. Impaired glucose regulation (IGR) was defined as $5.6 \leq \mathrm{FPG}<7.0$ $\mathrm{mmol} / \mathrm{L}$ and/or $7.8 \leq \mathrm{PPG}<11.1 \mathrm{mmol} / \mathrm{L}$. Hypertension was defined by a reported history thereof, SBP $\geq 140$ $\mathrm{mmHg}$, or DBP $\geq 90 \mathrm{mmHg}$, whereas prehypertension was defined by $120 \leq \mathrm{SBP}<140 \mathrm{mmHg}$ or $80 \leq \mathrm{DBP}$ $<90 \mathrm{mmHg}$. Patients were defined as suffering from dyslipidemia as per the Joint Committee for Developing Chinese Guidelines on Prevention and Treatment of Dyslipidemia in Adults criteria or based upon a selfreported history of hyperlipidemia. ${ }^{15}$ CVD was defined by self-reported history of cardiovascular events or conditions including myocardial infarction, stroke, and coronary heart disease. Any family history of cancer/T2DM was established using self-report questionnaires. The immunoturbidimetric method was used to measure urinary albumin, and the creatinine oxidase method was used to measure urinary creatinine. UACR was calculated based upon the ratio of urinary albumin to urinary creatinine, and was reported as a categorical variable $(\geq 30 \mathrm{mg} / \mathrm{g}$ or $<30 \mathrm{mg} / \mathrm{g}$ ). The primary outcome for the present study was an increased UACR $(\geq 30 \mathrm{mg} / \mathrm{g})$.

Participant age of natural menopause was defined based upon self-reported questionnaires, and women were defined as being postmenopausal if they had not experienced a menstrual period within $\geq 12$ months. ${ }^{16}$ As this study only included women who had experienced natural menopause, menopausal age was calculated as either the self-reported age at final menstrual period or the year final menstrual period minus birth year when subjects were unable to recall their exact age at time of menopause. These women were stratified into three groups based upon whether they experienced early, 
normal, or late menopause $(\leq 45,46-50$, and $>50$ years, respectively). ${ }^{17}$

\section{Statistical Analyses}

SPSS 22.0 (IBM, IL, USA) was employed for all statistical testing. The Kolmogorov-Smirnov test was employed to the normality of study data. Continuous data were given as mean $\pm \mathrm{SD}$ and median (interquartile range) when normally and non-normally distributed, respectively, whereas categorical variables are given as percentages. Continuous variables were compared via one-way ANOVAs or Kruskal-Wallis H-tests, while Pearson Chi-squared or Fisher's exact tests were used to compare categorical data as appropriate. Analytical models were assessed for potential multicollinearity, but no evidence of such collinearity was detected as evidenced by a variance inflation factor $<5$. Odds ratios (ORs) and $95 \%$ confidence intervals (CIs) were estimated using logistic regression analyses in order to assess the link between UACR and age at onset of natural menopause. Three different models were developed in an effort to appropriately adjust these analyses for potential confounding variables. Model 1 was adjusted for age and age at menarche. Model 2 was additionally adjusted for education level, sleep quality, CVD history, medication usage (hormone therapy, antihypertensive drugs, and hypoglycemic drugs), eGFR, and family history of cancer/diabetes. Model 3 was additionally adjusted for BMI, WC/HC, FBG, HDL-c, LDL-c, insulin, HbA1c, SBP, DBP, TG, ALT, AST, PBG, and GGT.

Subgroup analyses were conducted based upon patient age $(<60$ years, $\geq 60$ years $)$, BMI $\left(<24 \mathrm{~kg} / \mathrm{m}^{2}\right.$, 24-28 kg/m $\left.\mathrm{m}^{2}, \geq 28 \mathrm{~kg} / \mathrm{m}^{2}\right),{ }^{18}$ eGFR $(<90 \mathrm{~mL} /$ $\left.\left(\min \cdot 1.73 \mathrm{~m}^{2}\right), \geq 90 \mathrm{~mL} /\left(\min \cdot 1.73 \mathrm{~m}^{2}\right)^{19}\right)$, blood glucose, and BP as in prior studies. Interactions between age of menopause and potential confounding variables were assessed among different UACR groups. A two-sided $P<0.05$ was the significance threshold.

\section{Results}

\section{Study Participant Characteristics}

In total, 21,672 postmenopausal women were enrolled in this analysis and were stratified based upon whether they experienced early, normal, or late menopause $(\leq 45,46-50$, or $>50$ years, respectively; Table 1 ). The median baseline patient age was 59 (interquartile range: 55-65), and the median menopausal age of these participants was 50 (interquartile range: 48-52). Of these patients, 13.1\% $(\mathrm{n}=2846), 49.9 \%(\mathrm{n}=10,809)$, and $37.0 \%(\mathrm{n}=8017)$ experienced early, normal, and late menopause, respectively. More advanced menopausal age was associated with increased BMI $(P<0.0001)$, WC $(P<0.0001)$, BP $(P<0.0001)$, LDL-c $(P<0.0001)$, eGFR $(P=0.0027)$, hypertension incidence $(P=0.0003)$, and family history of cancer/diabetes $(P<0.0001)$. Earlier age of menopause, in contrast, was associated with a lower level of education $(P<0.0001)$ and with poorer quality of sleep $(P=0.0478)$. A higher frequency of women in the early menopause cohort exhibited a UACR $\geq 30 \mathrm{mg} / \mathrm{g}$ (Figure 2).

\section{The Relationship Between Age of Menopause Onset and Risk of Elevated UACR}

We next conducted a multifactor analysis to identify variables linked to elevated UACR in our study cohort, revealing BP, blood glucose, age, eGFR, BMI, and menopausal age to all be significantly linked to UACR (all $P<0.05$ ) (Table 2). In a subsequent logistic regression analysis, early menopause ( $\leq 45$ years) was linked to a higher risk of elevated UACR relative to normal menopause (46-50 years) (OR: 1.18, 95\% CI: 1.06-1.31), whereas late menopause ( $>50$ years) was linked to the opposite phenotype (OR: 0.87, 95\% CI: 0.80-0.94) (Table 3). After adjusting for a range of confounding variables, this relationship between menopausal age and risk of UACR elevation was still robust in all three of our statistical models $(\leq 45$ years: OR: $1.18,95 \%$ CI: $1.04-1.33$; $>50$ years: OR:0.86, 95\% CI: 0.78-0.94). Specifically, we determined that every 1-year rise in menopausal age was linked to a $3 \%$ reduction in risk of UACR elevation (OR: $0.97,95 \% \mathrm{CI}$ : 0.96-0.98).

\section{The Relationship Between Menopausal Age and Risk of Increased UACR in Participant Subgroups}

Multivariate adjusted ORs for women $\geq 60$ years old that experienced early or late menopause were 1.18 (95\% CI: 1.00-1.38) and 0.87 (95\% CI: 0.77-0.98), respectively. Similarly, the link between early menopause and increased was UACR was more robust in women who were obese (BMI $\geq 28 \mathrm{~kg} / \mathrm{m}^{2}$, OR: $1.52,95 \%$ CI: 1.13-2.04), had a lower eGFR ( $\leq 90$, OR: $1.22,95 \%$ CI: $1.04-1.44)$, were prediabetic $(5.6 \leq \mathrm{FPG}<7.0 \mathrm{mmol} / \mathrm{L}$ or $7.8 \leq \mathrm{PPG}<11.1$ $\mathrm{mmol} / \mathrm{L}$, OR: $1.27,95 \% \mathrm{CI}: 1.05-1.53$ ), or hypertensive (SBP $\geq 140 \mathrm{mmHg}$ or DBP $\geq 90 \mathrm{mmHg}$, OR: $1.31,95 \%$ 
Table I Characteristics of Participants Categorized by Age at Natural Menopause

\begin{tabular}{|c|c|c|c|c|c|}
\hline Characteristics & Total & $\leq 45$ y $(n=2846)$ & $45-50$ y $(n=10,809)$ & $>50$ y $(n=80 \mid 7)$ & $P$ value \\
\hline Age $(y)$ & $59.14(55.10-65.37)$ & $60.12(54.82-67.79)$ & $58.89(54.50-65.63)$ & $59.10(55.82-64.32)$ & $<0.0001$ \\
\hline Age at menarche $(y)$ & $15.00(\mid 4.00-17.00)$ & $15.00(\mid 4.00-17.00)$ & $15.00(\mid 4.00-17.00)$ & $15.00(\mid 4.00-17.00)$ & $<0.0001$ \\
\hline Menopausal age (y) & $50.00(48.00-52.00)$ & $44.00(42.00-45.00)$ & $49.00(48.00-50.00)$ & $53.00(52.00-54.00)$ & $<0.0001$ \\
\hline BMI $\left(\mathrm{kg} / \mathrm{m}^{2}\right)$ & $24.22(22.03-26.58)$ & $24.13(21.83-26.61)$ & $24.12(21.93-26.44)$ & $24.56(22.38-26.89)$ & $<0.0001$ \\
\hline$W C(\mathrm{~cm})$ & $85.00(78.30-92.00)$ & $85.00(78.20-92.03)$ & $85.00(78.20-91.50)$ & $86.00(80.00-93.00)$ & $<0.0001$ \\
\hline $\mathrm{WC} / \mathrm{HC}$ & $0.88(0.84-0.92)$ & $0.88(0.84-0.93)$ & $0.88(0.84-0.92)$ & $0.88(0.84-0.93)$ & $<0.0001$ \\
\hline SBP $(m m H g)$ & $130.67(\mid 18.00-145.67)$ & $129.33(\mid 16.00-144.67)$ & $130.00(\mid 17.67-145.00)$ & $132.00(|| 9.33-146.67)$ & $<0.0001$ \\
\hline $\mathrm{DBP}(\mathrm{mmHg})$ & $75.67(69.33-83.00)$ & $74.67(68.67-82.00)$ & $75.67(69.33-82.67)$ & $76.00(69.67-83.33)$ & $<0.0001$ \\
\hline FBG $(\mathrm{mmol} / \mathrm{L})$ & $5.54(5.12-6.16)$ & $5.59(5.17-6.20)$ & $5.54(5.12-6.15)$ & $5.55(5.13-6.17)$ & 0.0061 \\
\hline PBG (mmol/L) & $7.59(6.20-9.94)$ & $7.80(6.30-10.46)$ & $7.59(6.20-9.90)$ & $7.43(6.14-9.80)$ & 0.0001 \\
\hline $\mathrm{HbAlc}(\%)$ & $6.00(5.70-6.30)$ & $6.00(5.70-6.40)$ & $5.90(5.70-6.30)$ & $6.00(5.70-6.30)$ & 0.0237 \\
\hline Insulin $(\mu \mathrm{U} / \mathrm{mL})$ & $7.60(5.50-10.50)$ & $7.70(5.70-10.70)$ & $7.60(5.50-10.50)$ & $7.80(5.70-10.70)$ & 0.0002 \\
\hline TG (mmol/L) & $1.40(1.01-1.99)$ & $1.44(1.02-2.04)$ & $1.4 I(1.02-2.01)$ & $1.44(1.05-2.03)$ & 0.0328 \\
\hline LDL-c (mmol/L) & $3.04(2.45-3.66)$ & $2.90(2.31-3.56)$ & $3.05(2.48-3.66)$ & $3.14(2.56-3.74)$ & $<0.0001$ \\
\hline HDL-c (mmol/L) & $1.34(1.14-1.57)$ & $1.32(1.11-1.53)$ & $1.35(1.15-1.57)$ & $1.35(1.15-1.57)$ & $<0.0001$ \\
\hline ALT (mmol/L) & $15.00(11.00-20.00)$ & $14.00(10.00-20.00)$ & $14.00(11.00-20.00)$ & $15.00(11.00-21.00)$ & 0.0187 \\
\hline AST (mmol/L) & $20.00(17.00-25.00)$ & $21.00(17.00-25.00)$ & $21.00(17.00-25.00)$ & $21.00(18.00-25.00)$ & $<0.0001$ \\
\hline GGT (mmol/L) & $19.00(14.00-28.00)$ & $19.00(14.00-28.00)$ & $19.00(14.00-29.00)$ & $20.00(15.00-29.00)$ & $<0.0001$ \\
\hline $\mathrm{eGFR}\left(\mathrm{mL} / \mathrm{min} / \mathrm{I} .73 \mathrm{~m}^{2}\right)$ & & & & & 0.0027 \\
\hline$>90$ & II,789(54.44\%) & $1480(52.08 \%)$ & $5848(54.13 \%)$ & $446 \mid(55.69 \%)$ & \\
\hline$\leq 90$ & $9867(45.56 \%)$ & $1362(47.92 \%)$ & $4955(45.87 \%)$ & $3550(44.31 \%)$ & \\
\hline Education (\%) & & & & & $<0.0001$ \\
\hline Low & $5208(24.14 \%)$ & $854(30.16 \%)$ & $2663(24.75 \%)$ & $169 \mid(21.17 \%)$ & \\
\hline Intermediate & $14,526(67.32 \%)$ & $1797(63.45 \%)$ & $7254(67.42 \%)$ & $5475(68.56 \%)$ & \\
\hline High & I844(8.55\%) & $|8|(6.39 \%)$ & $843(7.83 \%)$ & $820(10.27 \%)$ & \\
\hline Sleep quality (\%) & & & & & 0.0478 \\
\hline Good & $|6,62|(76.69 \%)$ & $2151(75.58 \%)$ & $8252(76.34 \%)$ & $6218(77.56)$ & \\
\hline Poor & $503 \mid(23.31 \%)$ & $695(24.42 \%)$ & $2557(23.66 \%)$ & $1799(22.44 \%)$ & \\
\hline Smoking status (\%) & & & & & 0.3053 \\
\hline No smoking & $21,105(98.23 \%)$ & $10,528(98.16 \%)$ & 2749(97.97) & $7828(98.40 \%)$ & \\
\hline Smoking & $324(1.51 \%)$ & $163(1.52 \%)$ & $5 \mathrm{I}(\mathrm{I} .82 \%)$ & $110(1.38 \%)$ & \\
\hline Quit smoking & $57(0.27 \%)$ & $34(0.32 \%)$ & $6(0.21 \%)$ & $17(0.21 \%)$ & \\
\hline
\end{tabular}


Table I (Continued).

\begin{tabular}{|c|c|c|c|c|c|}
\hline Characteristics & Total & $\leq 45$ y $(n=2846)$ & $45-50$ y $(n=10,809)$ & $>50$ y $(n=8017)$ & $P$ value \\
\hline Drinking status (\%) & & & & & 0.6932 \\
\hline No drinking & $19,035(88.52 \%)$ & $2488(88.45 \%)$ & $9485(88.46 \%)$ & $7062(88.63 \%)$ & \\
\hline Drinking & $2400(11.16 \%)$ & $320(11.38 \%)$ & $120 \mid(\mid 1.20 \%)$ & $879(11.03 \%)$ & \\
\hline Quit drinking & $68(0.32 \%)$ & $5(0.18 \%)$ & $36(0.34 \%)$ & $27(0.34 \%)$ & \\
\hline \multicolumn{6}{|l|}{ Prevalence of diseases (\%) } \\
\hline Diabetes mellitus & $2618(12.13 \%)$ & $400(14.13 \%)$ & $1253(11.64 \%)$ & $965(12.07 \%)$ & 0.0014 \\
\hline Hypertension & $5268(25.12 \%)$ & $630(23.23 \%)$ & $2562(24.52 \%)$ & $2076(26.58 \%)$ & 0.0003 \\
\hline Dyslipidemia & $2126(10.22 \%)$ & $264(9.83 \%)$ & $986(9.52 \%)$ & $876(11.29 \%)$ & 0.0004 \\
\hline CVD & $1080(5.19 \%)$ & $172(6.39 \%)$ & $508(4.91 \%)$ & $400(5.16 \%)$ & 0.0085 \\
\hline Tumor & $649(3.02 \%)$ & $99(3.52 \%)$ & $324(3.02 \%)$ & $226(2.84 \%)$ & 0.1943 \\
\hline \multicolumn{6}{|l|}{ Medication (\%) } \\
\hline Antihypertensive drugs & $3379(15.6 \%)$ & $382(13.4 \%)$ & $1622(15.0 \%)$ & $1375(17.2 \%)$ & $<0.0001$ \\
\hline Hypoglycemic drugs & $2191(18.98 \%)$ & $328(20.10 \%)$ & $1050(18.00 \%)$ & $813(19.92 \%)$ & 0.0261 \\
\hline Lipid-lowering drugs & $99(0.46 \%)$ & $10(0.35 \%)$ & $47(0.43 \%)$ & $42(0.52 \%)$ & 0.4484 \\
\hline Hormone therapy & $798(4 \%)$ & $115(4.4 \%)$ & $400(4.1 \%)$ & $283(3.9 \%)$ & 0.5423 \\
\hline \multicolumn{6}{|l|}{ Family history (\%) } \\
\hline Family history of diabetes & $3722(17.33 \%)$ & $410(14.54 \%)$ & $1789(\mid 6.70 \%)$ & $1523(19.15 \%)$ & $<0.0001$ \\
\hline Family history of tumor & $3201(14.9 \%)$ & $361(12.81 \%)$ & $1545(\mid 4.43 \%)$ & $1295(\mid 6.29 \%)$ & $<0.0001$ \\
\hline
\end{tabular}

Note: The data were expressed as median (interquartile range) for skewed distributed continuous variables and numbers (percentages) for categorical variables. Abbreviations: BMI, body mass index; WC, waist circumference; WC/HC, waist-to-hip ratio; SBP, systolic blood pressure; DBP, diastolic blood pressure; FBG, 0-hour fasting blood glucose; PBG, 2-hour postprandial blood glucose; HbAlc, glycated hemoglobin; TG, triglyceride; LDL-c, low-density lipoprotein cholesterol; HDL-c, highdensity lipoprotein cholesterol; ALT, alanine aminotransferase; AST, aspartate aminotransferase; GGT, gamma-glutamyltranspeptidase; eGFR, estimated glomerular filtration rate; CVD, cardiovascular diseases.

CI: 1.09-1.57). The link between late menopause and lower rates of UACR elevation was more robust for women who exhibited a normal weight $\left(\mathrm{BMI}<24 \mathrm{~kg} / \mathrm{m}^{2}\right.$, OR: $0.78,95 \%$ CI: $0.67-0.89$ ), normal BP (SBP $<120$ and DBP $<80 \mathrm{mmHg}$, OR: $0.73,95 \%$ CI: 0.58-0.91), normal blood glucose (FBG $<5.6 \mathrm{mmol} / \mathrm{L}$ and $\mathrm{PBG}<7.8 \mathrm{mmol} / \mathrm{L}$, OR: $0.82,95 \%$ CI: $0.69-0.96$ ), or had diabetes (FBG $\geq 7.0$ $\mathrm{mmol} / \mathrm{L}$ or $\mathrm{PBG} \geq 11.1 \mathrm{mmol} / \mathrm{L}$, OR: $0.79,95 \%$ CI: $0.67-$ 0.94). No interactions among any of these variables were observed (Figure 3).

\section{Discussion}

Overall, the present cross-sectional analyses revealed that early menopause ( $\leq 45$ years old) was linked to a significantly higher risk of having an elevated UACR, whereas the opposite was true for late menopause $(>50$ years old). This relationship was significant even following adjustment for a wide range of confounding variables and after conducting subgroup analyses based upon participant age, BMI, BP, blood glucose, and eGFR. This is the first large-scale multi-center study to our knowledge to have surveyed the relationship between menopausal age and UACR among postmenopausal Chinese women.

Menopausal age has also been linked to DNA damage repair, ${ }^{20,21}$ such that earlier menopause onset may be indicative of accelerated somatic aging and a predictor of overall health status during later life. ${ }^{22}$ Multiple analyses to date have explored the association between menopausal age and cardiovascular disease-related outcomes. For example, Muka et $\mathrm{al}^{4}$ conducted a meta-analysis of 32 studies (310,000 women), revealing early menopause ( $<45$ years) to be linked to a higher risk of coronary artery disease relative to menopause onset at 45 years of age or 


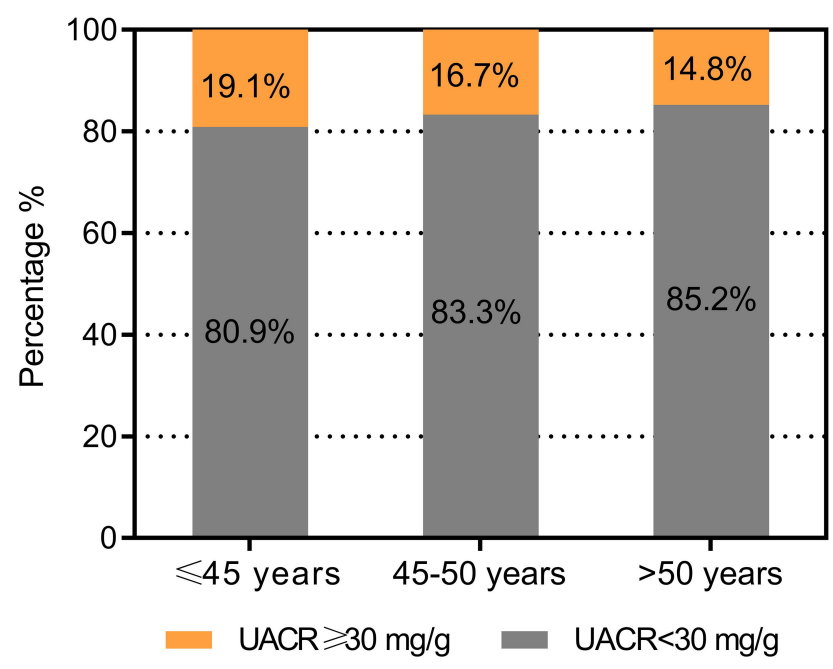

Figure 2 Percentage of urinary albumin-creatinine ratio (UACR) levels at diverse nature menopausal ages.

older. Similarly, another study observed elevated cardiovascular risk among those who experienced premature menopause even after adjusting for other risk factors. ${ }^{23}$ Early menopause may thus increase cardiovascular risk, rather than merely being a consequence of such risk. ${ }^{2}$ Microalbuminuria is a key biomarker of renal vascular damage and an important predictor of CVD. ${ }^{24}$ Few studies to date, however, have explored the potential relationship between menopausal age and microalbuminuria as a metric for CKD risk. In one study of postmenopausal women in South Korea, the prevalence of proteinuria fell with increasing reproductive period such that it was lowest $(2.3 \%)$ when this period was $>45$ years. ${ }^{25}$

There may be a number of mechanistic explanations for the observed relationship between age at menopause onset and microalbuminuria risk. Menopause is associated with an up to $80 \%$ reduction in estrogen levels, and the early loss of such estrogen may result in prolonged RAS activation, inflammation, immunological/ endothelial dysfunction, and consequent damage to the vascular system. ${ }^{26,27}$ Such dysregulation may be facilitated in part by the transmembrane $G$ protein-coupled estrogen receptor. ${ }^{27}$ Estrogen signaling can also protect kidney cells from oxidative damage and suppress renal superoxide generation, ${ }^{28}$ such that postmenopausal women exhibit higher levels of oxidative stress and inflammatory cytokine production. ${ }^{29}$ Oxidative stress can drive the apoptotic death of podocytes, causing glomerular sclerosis and signal transduction that can result in interstitial fibrosis as a consequence of the secretion of renal tubular extracellular matrix factors. ${ }^{30}$ Kattah et al ${ }^{31}$
Table 2 Multiple Factors Related to UACR

\begin{tabular}{|c|c|c|}
\hline & OR (95\% CI) & $P$ value \\
\hline Menopausal age (y) & & $<0.0001$ \\
\hline$\leq 45$ & I.II (0.99-I.25) & \\
\hline $45-50$ & 1.00 & \\
\hline$>50$ & $0.86(0.79-0.94)$ & \\
\hline $\mathrm{BP}(\mathrm{mmHg})$ & & $<0.0001$ \\
\hline SBP $<120$ and $D B P<80$ & 1.00 & \\
\hline $\begin{array}{l}\quad 120 \leq S B P<140 \text { and } / \text { or } 80 \leq \mathrm{DBP} \\
<90\end{array}$ & $1.09(0.97-1.22)$ & \\
\hline$S B P \geq 140$ or $D B P \geq 90$ & $1.78(1.60-1.99)$ & \\
\hline Blood glucose(mmol/L) & & $<0.0001$ \\
\hline FBG $<5.6$ and $P B G<7.8$ & 1.00 & \\
\hline $5.6 \leq \mathrm{FBG}<7.0$ or $7.8 \leq \mathrm{PBG}<1 \mathrm{I} .1$ & $1.12(1.02-1.23)$ & \\
\hline$F B G \geq 7.0$ or $P B G \geq 11.1$ & $1.67(1.50-1.85)$ & \\
\hline $\operatorname{eGFR}\left(\mathrm{mL} / \mathrm{min} / 1.73 \mathrm{~m}^{2}\right)$ & & $<0.0001$ \\
\hline$>90$ & 1.00 & \\
\hline$\leq 90$ & $1.22(1.11-1.33)$ & \\
\hline Age $(y)$ & $1.03(1.03-1.04)$ & $<0.0001$ \\
\hline HDL-c (mmol/L) & $0.72(0.63-0.83)$ & $<0.0001$ \\
\hline LDL-c (mmol/L) & $0.80(0.76-0.84)$ & $<0.0001$ \\
\hline $\mathrm{TG}(\mathrm{mmol} / \mathrm{L})$ & $1.09(1.06-1.13)$ & $<0.0001$ \\
\hline AST (mmol/L) & $1.00(1.00-1.01)$ & 0.0085 \\
\hline GGT (mmol/L) & $1.00(1.00-1.00)$ & 0.0002 \\
\hline $\mathrm{BMI}\left(\mathrm{kg} / \mathrm{m}^{2}\right)$ & $1.03 I(I .022-1.04 I)$ & $<0.0001$ \\
\hline
\end{tabular}

Abbreviations: UACR, urinary albumin-creatinine ratio; SBP, systolic blood pressure; DBP, diastolic blood pressure; FBG, 0-hour fasting blood glucose; PBG, 2-hour postprandial blood glucose; eGFR, estimated glomerular filtration rate; LDL-c, lowdensity lipoprotein cholesterol; HDL-c, high-density lipoprotein cholesterol; TG, triglyceride; AST, aspartate aminotransferase; GGT, gamma-glutamyltranspeptidase; BMI, body mass index.

found that hormone replacement therapy was associated with a lower risk of UACR in a meta-analysis. In light of this association, we adjusted for hormone therapy in the present analysis. Large-scale genome-wide association studies have further led to the identification of multiple genetic loci associated with age of menopause onset that were associated with genome stability, inflammation, and oxidative stress. ${ }^{32}$ As such, both environmental and genetic risk factors may drive the incidence of early menopause, ${ }^{33}$ which in turn represents an important biomarker of elevated risk of a range of adverse health 
Table 3 Adjusted Odds Ratios (95\% Confidence Intervals) for UACR According to Age at Natural Menopause

\begin{tabular}{|c|c|c|c|c|c|c|c|c|}
\hline \multirow[t]{2}{*}{ Menopausal Age } & \multicolumn{2}{|l|}{ Nonadjusted } & \multicolumn{2}{|l|}{ Model I } & \multicolumn{2}{|l|}{ Model 2} & \multicolumn{2}{|l|}{ Model 3} \\
\hline & OR (95\% CI) & $P$ value & OR (95\% Cl) & $P$ value & OR (95\% Cl) & $P$ value & OR (95\% Cl) & $P$ value \\
\hline All subjects & & $<0.0001$ & & $<0.0001$ & & $<0.0001$ & & $<0.0001$ \\
\hline$\leq 45$ y & $1.18(1.06-1.31)$ & 0.0027 & I.14(I.02-1.27) & 0.0205 & $1.19(1.06-1.34)$ & 0.0025 & $1.18(1.04-1.33)$ & 0.0097 \\
\hline $45-50$ y & 1.00 & & 1.00 & & 1.00 & & 1.00 & \\
\hline$>50 y$ & $0.87(0.80-0.94)$ & 0.0006 & $0.87(0.80-0.94)$ & 0.0008 & $0.85(0.78-0.93)$ & 0.0004 & $0.86(0.78-0.94)$ & 0.0009 \\
\hline $\begin{array}{l}\text { Continuous menopausal } \\
\text { age }\end{array}$ & $0.98(0.97-0.99)$ & $<0.0001$ & $0.97(0.97-0.98)$ & $<0.0001$ & $0.97(0.96-0.98)$ & $<0.0001$ & $0.97(0.96-0.98)$ & $<0.0001$ \\
\hline
\end{tabular}

Notes: Nonadjusted model. Model I was adjusted for age and age at menarche; model 2 was additionally adjusted for education level, sleep quality, cardiovascular history, medication usage (hormone therapy, antihypertensive drugs, and hypoglycemic drugs), eGFR, and family history of cancer/diabetes; model 3 was additionally adjusted for BMI, WC/HC, FBG, PBG, insulin, HbAIc, SBP, DBP, TG, LDL-c, HDL-c, AST, ALT, and GGT.

outcomes. The observed link between age at natural menopause onset and increased risk of microalbuminuria in the present study may be attributable to any of the above mechanisms.

Obesity is often correlated with greater insulin resistance, which has the potential to harm renal podocytes and thereby disrupt renal hemodynamics. Herein, we found menopausal age and UACR to be more strongly correlated with one another in obese women relative to women with lower BMI values. Risk of UACR elevation was also significantly higher among women who experienced early menopause with an eGFR $\leq 90 \mathrm{~mL} / \mathrm{min}$ per $1.73 \mathrm{~m}^{2}$, prediabetes, or hypertension. Appropriate screening and preventative measures should therefore be taken by these women.

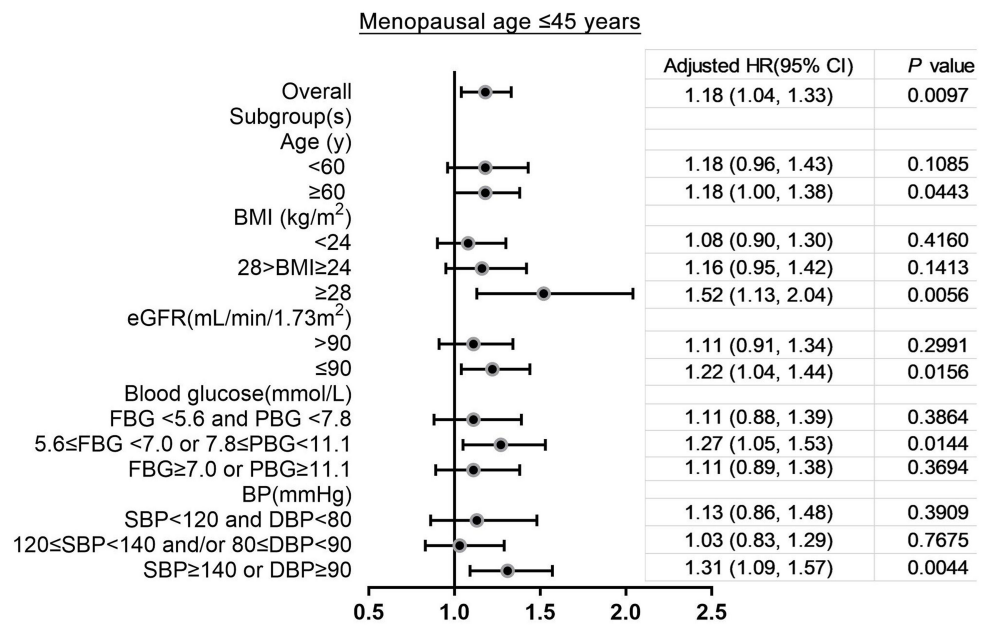

\section{Limitations and Future Directions}

This study has a number of strengths. For one, it was a large-scale study of representative populations from seven different regions. In addition, we adjusted our statistical models for many different potential confounding factors and explored the relationship between age of menopause and UACR in a range of different subgroups. However, there are multiple limitations to this study. First, as age of menopause onset was established using selfreported questionnaires, our data are potentially susceptible to recall bias. However, prior work suggests that the validity and reproducibility of self-reported age of menopause are generally satisfactory. ${ }^{34,35}$ Second, we are unable to exclude the possibility that other confounding factors may have influenced the analyzed relationships in

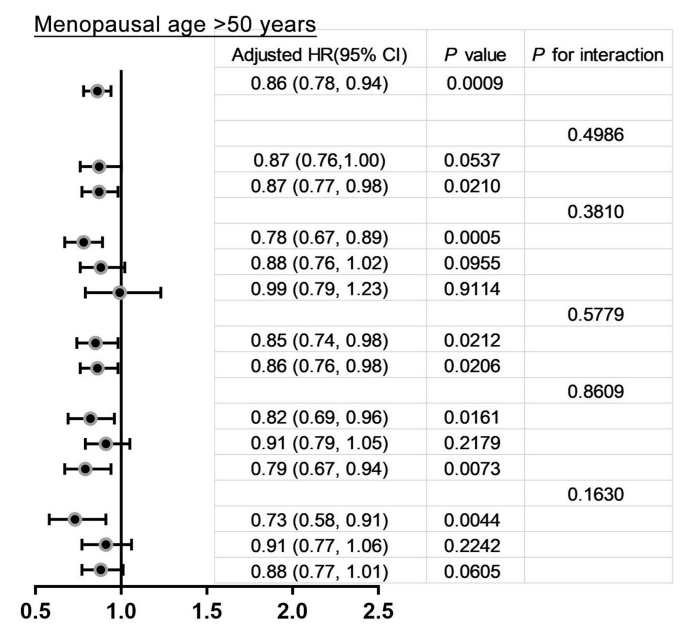

Figure 3 Odds ratios for logistic regression model 3 of the association between nature menopausal ages and urinary albumin-creatinine ratio (UACR) in postmenopausal women with different subgroups.

Abbreviations: BMI, body mass index; eGFR, estimated glomerular filtration rate; FBG, 0-hour fasting blood glucose; PBG, 2-hour postprandial blood glucose; BP, blood pressure; SBP, systolic blood pressure; DBP, diastolic blood pressure. 
the present study, such as estrogen levels or parity. ${ }^{36}$ Third, urinary albumin levels were measured using a single urine sample collected in the morning, whereas measuring multiple samples would have provided greater result stability. That being said, individual spot urine samples have been shown to correlate well with the results of multiple or 24 $\mathrm{h}$ urine sample collection protocols, suggesting that this single-sample approach is sufficient for large-scale epidemiological studies. ${ }^{37}$ As this was a cross-sectional study, we were also not able to draw any conclusions with respect to causality. Future prospective studies will be essential in order to understand the mechanistic link between microalbuminuria and age of menopause onset.

\section{Conclusion}

In conclusion, early-onset menopause ( $\leq 45$ years) was linked to a significantly higher microalbuminuria risk in postmenopausal women. Further work will be required to explore the mechanistic basis for this relationship and to understand whether the timing of menopause can offer value as a means of predicting and preventing microalbuminuria. Research is needed to understand the mechanisms underlying these associations and explore whether the timing of natural menopause can add value to microalbuminuria prediction and prevention.

\section{Data Sharing Statement}

All data used to support the findings of this study are available from the corresponding author upon request.

\section{Acknowledgments}

This work is part of the REACTION study. The authors would like to thank all the reviewers who participated in the review and MJEditor for its linguistic assistance during the preparation of this manuscript.

\section{Funding}

The study is supported by the Chinese Society of Endocrinology, the Key Laboratory for Endocrine and Metabolic Diseases of Ministry of Health (1994DP131044), the National Key New Drug Creation and Manufacturing Program of Ministry of Science and Technology (2012ZX09303006-001), the National High Technology Research and Development Program of China (863 Program, 2011AA020107), the National Natural Science Foundation of China (81300717), and the National Science and Technology Major Project 288 (2011ZX09307-001-8).

\section{Disclosure}

The authors report no conflicts of interest.

\section{References}

1. Snowdon DA, Kane RL, Beeson WL, et al. Is early natural menopause a biologic marker of health and aging? Am J Public Health. 1989;79(6):709-714. doi:10.2105/AJPH.79.6.709

2. Manson JE, Woodruff TK. Reproductive health as a marker of subsequent cardiovascular disease: the role of estrogen. JAMA Cardiology. 2016;1(7):776-777. doi:10.1001/jamacardio.2016.2662

3. Velez MP, Alvarado BE, Rosendaal N, et al. Age at natural menopause and physical functioning in postmenopausal women: the Canadian Longitudinal Study on Aging. Menopause. 2019;26 (9):958-965. doi:10.1097/GME.0000000000001362

4. Muka T, Oliver-Williams C, Kunutsor S, et al. Association of age at onset of menopause and time since onset of menopause with cardiovascular outcomes, intermediate vascular traits, and all-cause mortality: a systematic review and meta-analysis. JAMA Cardiol. 2016;1 (7):767-776. doi:10.1001/jamacardio.2016.2415

5. Mikhail A, Brown C, Williams JA, et al. Renal association clinical practice guideline on Anaemia of Chronic Kidney Disease. BMC Nephrol. 2017;18(1):345. doi:10.1186/s12882-017-0688-1

6. Fujii H, Kono K, Nishi S. Characteristics of coronary artery disease in chronic kidney disease. Clin Exp Nephrol. 2019;23(6):725-732. doi:10.1007/s10157-019-01718-5

7. Jensen JS, Borch-Johnsen K, Jensen G, Feldt-Rasmussen B. Microalbuminuria reflects a generalized transvascular albumin leakiness in clinically healthy subjects. Clin Sci. 1995;88(6):629-633. doi:10.1042/cs0880629

8. Konno S, Munakata M, Reboldi G. Moderately increased albuminuria is an independent risk factor of cardiovascular events in the general Japanese population under 75 years of age: the Watari study. PLoS One. 2015;10(4):e0123893. doi:10.1371/journal.pone.0123893

9. Scirica BM, Mosenzon O, Bhatt DL, et al. Cardiovascular outcomes according to urinary albumin and kidney disease in patients with type 2 diabetes at high cardiovascular risk: observations from the SAVOR-TIMI 53 Trial. JAMA Cardiol. 2018;3(2):155-163. doi:10. 1001/jamacardio.2017.4228

10. Marquez DF, Ruiz-Hurtado G, Segura J, Ruilope L. Microalbuminuria and cardiorenal risk: old and new evidence in different populations. F1000Research. 2019;8:8. doi:10.12688/ f1000research.17212.1

11. Matsushita K, Van Der Velde M; Chronic Kidney Disease Prognosis C. Association of estimated glomerular filtration rate and albuminuria with all-cause and cardiovascular mortality in general population cohorts: a collaborative meta-analysis. Lancet. 2010;375(9731): 2073-2081.

12. Valmadrid CT, Klein R, Moss SE, Klein BEK. The risk of cardiovascular disease mortality associated with microalbuminuria and gross proteinuria in persons with older-onset diabetes mellitus. Arch Intern Med. 2000;160(8):1093-1100. doi:10.1001/archinte.160.8.1093

13. Bi Y, Lu J, Wang W, et al. Cohort profile: risk evaluation of cancers in Chinese diabetic individuals: a longitudinal (REACTION) study. $J$ Diabetes. 2014;6(2):147-157. doi:10.1111/1753-0407.12108

14. Levey AS, Stevens LA, Schmid $\mathrm{CH}$, et al. A new equation to estimate glomerular filtration rate. Ann Intern Med. 2009;150 (9):604-612. doi:10.7326/0003-4819-150-9-200905050-00006

15. Joint committee for guideline r. 2016 Chinese guidelines for the management of dyslipidemia in adults. $J$ Geriatric Cardiol. 2018;15 (1):1-29. doi:10.11909/j.issn.1671-5411.2018.01.011

16. Harlow SD, Gass M, Hall JE, et al. Executive summary of the stages of reproductive aging workshop +10 : addressing the unfinished agenda of staging reproductive aging. J Clin Endocrinol Metab. 2012;97(4):1159-1168. doi:10.1210/jc.2011-3362 
17. Jiang J, Cui J, Wang A, et al. Association Between Age at Natural Menopause and Risk of Type 2 Diabetes in Postmenopausal Women With and Without Obesity. J Clin Endocrinol Metab. 2019;104 (7):3039-3048. doi:10.1210/jc.2018-02310

18. Zhou B-F. Predictive values of body mass index and waist circumference for risk factors of certain related diseases in Chinese adults study on optimal cut-off points of body mass index and waist circumference in Chinese adults. Biomed Environ Sci. 2002;15 (1):83-96.

19. Khwaja A. KDIGO clinical practice guidelines for acute kidney injury. Nephron Clin Pract. 2012;120(4):c179-c184. doi:10.1159/ 000339789

20. Jackson SP, Bartek J. The DNA-damage response in human biology and disease. Nature. 2009;461(7267):1071-1078. doi:10.1038/nature 08467

21. Day FR, Ruth KS, Thompson DJ, et al. Large-scale genomic analyses link reproductive aging to hypothalamic signaling, breast cancer susceptibility and BRCA1-mediated DNA repair. Nat Genet. 2015;47(11):1294-1303. doi:10.1038/ng.3412

22. Laven JSE, Visser JA, Uitterlinden AG, Vermeij WP, Hoeijmakers JHJ. Menopause: genome stability as new paradigm. Maturitas. 2016;92:15-23. doi:10.1016/j.maturitas.2016.07.006

23. Honigberg MC, Zekavat SM, Aragam K, et al. Association of premature natural and surgical menopause with incident cardiovascular disease. JAMA. 2019;18.

24. Yudkin JS, Forrest RD, Jackson CA. Microalbuminuria as predictor of vascular disease in non-diabetic subjects. Islington Diabetes Survey. Lancet. 1988;332(8610):530-533. doi:10.1016/S0140-6736 (88)92657-8

25. Noh JH, Koo H. Older menarche age and short reproductive period linked to chronic kidney disease risk. Medicine. 2019;98(18):e15511. doi:10.1097/MD.0000000000015511

26. Ruiz-Ortega M, Lorenzo O, Ruperez M, et al. Role of the renin-angiotensin system in vascular diseases: expanding the field. Hypertension. 2001;38(6):1382-1387. doi:10.1161/hy1201.100589

27. Zhao Z, Wang H, Jessup JA, Lindsey SH, Chappell MC, Groban L. Role of estrogen in diastolic dysfunction. Am J Physiol Heart Circ Physiol. 2014;306(5):H628-H640. doi:10.1152/ajpheart.00859.2013
28. Ji H, Zheng W, Menini S, et al. Female protection in progressive renal disease is associated with estradiol attenuation of superoxide production. Gend Med. 2007;4(1):56-71. doi:10.1016/S1550-8579 (07)80009-X

29. Paik JK, Kim JY, Kim OY, et al. Circulating and PBMC Lp-PLA2 associate differently with oxidative stress and subclinical inflammation in nonobese women (menopausal status). PLoS One. 2012;7(2): e29675. doi:10.1371/journal.pone.0029675

30. Ogura S, Shimosawa T. Oxidative stress and organ damages. Curr Hypertens Rep. 2014;16(8):452. doi:10.1007/s11906-014-0452-x

31. Kattah AG, Suarez MLG, Milic N, et al. Hormone therapy and urine protein excretion: a multiracial cohort study, systematic review, and meta-analysis. Menopause. 2018;25(6):625-634. doi:10.1097/GME. 0000000000001062

32. Stolk L, Perry JRB, Chasman DI, et al. Meta-analyses identify 13 loci associated with age at menopause and highlight DNA repair and immune pathways. Nat Genet. 2012;44(3):260-268. doi:10.1038/ ng. 1051

33. Rocca WA, Shuster LT, Grossardt BR, et al. Long-term effects of bilateral oophorectomy on brain aging: unanswered questions from the mayo clinic cohort study of oophorectomy and aging. Women's Health. 2009;5(1):39-48. doi:10.2217/17455057.5.1.39

34. den Tonkelaar I. Validity and reproducibility of self-reported age at menopause in women participating in the DOM-project. Maturitas. 1997;27(2):117-123. doi:10.1016/S0378-5122(97)01122-5

35. Cairns BJ, Liu B, Clennell S, et al. Lifetime body size and reproductive factors: comparisons of data recorded prospectively with self reports in middle age. BMC Med Res Methodol. 2011;11(1):7. doi:10.1186/1471-2288-11-7

36. Must A, et al. Recall of early menstrual history and menarcheal body size: after 30 years, how well do women remember? Am J Epidemiol. 2002;155(7):672-679. doi:10.1093/aje/155.7.672

37. Eknoyan G, Hostetter T, Bakris GL, et al. Proteinuria and other markers of chronic kidney disease: a position statement of the national kidney foundation (NKF) and the national institute of diabetes and digestive and kidney diseases (NIDDK). Am J Kidney Dis. 2003;42(4):617-622. doi:10.1016/S0272-6386(03)00826-6

\section{Publish your work in this journal}

Diabetes, Metabolic Syndrome and Obesity: Targets and Therapy is an international, peer-reviewed open-access journal committed to the rapid publication of the latest laboratory and clinical findings in the fields of diabetes, metabolic syndrome and obesity research. Original research, review, case reports, hypothesis formation, expert opinion and commentaries are all considered for publication. The manuscript management system is completely online and includes a very quick and fair peer-review system, which is all easy to use. Visit http://www.dovepress.com/testimonials.php to read real quotes from published authors.

Submit your manuscript here: https://www.dovepress.com/diabetes-metabolic-syndrome-and-obesity-targets-and-therapy-journal 